\title{
ASSESSMENT OF SPECIFICITY OF ANTISERA FOR IMMUNOHISTOCHEMISTRY
}

\author{
Katsumi WAKABAYASHI and Shigeyasu TANAKA \\ Institute of Endocrinology, Gunma University, 3-39-15, Showa-machi, \\ Maebashi, Gunma 371
}

\begin{abstract}
The problem of specificity of antisera is one of the most important points in immunohistochemistry as well as in radioimmunoassay (RIA). However, the evaluatiion about specificity required in these two methods are quite different. In immunohistochemical staining, antisera which can never be used in RIA have been successefully employed. For example, anti-hCG was used for staining rat pituitary LH cells, and anti-mammalian LHRH was used for staining avian hypothalamus. On the other hand, very useful antisera for RIA are often eliminated in immunohistochemistry because of poor specificity. Such different evaluation is understandable if we compare the principles of these methods. The principle of RIA is the competitive binding, while immunohistochemistry based on the non-competitive binding where substances with very low affinities to the antibody can be bound if the concentration of antibody is satisfactorily high.

In immunohistochemistry, the coexistence of antibodies to substances other than target antigen is very significant, while in RIA only the crossreactivity is important, and the competitive binding test gives no information about that. So, the evaluation of the antiserum in immunohistochemistry should be carried out using the non-competitive binding test which is based on the same principle to that of immunohistochemistry, and the results should be compared with those obtained with the competitive binding test which gives important information about the difference of the affinity constants of crossreacting substances.
\end{abstract}

The principle of RIA is the competitive binding of labeled and non-labeled antigens to a small amount of antibody. The effect of a crossreacting substance on the assay value is decided from the ratio of its association constant to that of the true antigen. On the other hand, the principle of immunohistochemistry is non-competitive binding of antigen and antibody where even a substance with comparatively poor affinity to the antibody can be stained, and the coexistence of an antibody to other antigenic substances critically effects on the specificity of the staining.

In the present paper, the authors wish to clarify the difference of antiserum evaluation in immunohistochemistry and RIA, and show the methods of assessment of antisera for immunohistochemistry. 


\section{MATERIALS AND METHODS}

\section{Radioiodination}

${ }^{125}$ I-labeled antigens were prepared according to the method of Hunter and Greenwood (3) with minor modification. The specific activity of the radioiodinated antigens was 50 to $100 \mu \mathrm{Ci} / \mu \mathrm{g}$.

Competitive binding tests

Competitive binding experiments were carried out according to a double antibody RIA procedure. The incubation system consisited of $300 \mu \mathrm{l}$ of BSA-PBS [phosphate-buffered saline $(0.01 \mathrm{M}$ sodium phosphate and $0.15 \mathrm{M} \mathrm{NaCl})$ containing $1 \%$ bovine serum albumin (Fraction $\mathrm{V}$ ) and $0.05 \% \mathrm{NaN}_{3}, \mathrm{pH} 7.5$ ], $100 \mu \mathrm{l}$ of various concentrations of the antigen or its analogues, $100 \mu \mathrm{l}$ of either $1 \%$ normal rabbit serum (NRS)-0.05 M EDTA-PBS, pH 7.5 or diluted antiserum in 1\% NRS-EDTAPBS, and $100 \mu \mathrm{l}$ of ${ }^{125}$ I-labeled antigen dissolved in BSA-PBS (about 20,000 cpm). The mixture was incubated at room temperature overnight, then added to $250 \mu \mathrm{l}$ of the second antibody (goat anti-rabbit IgG serum) diluted in EDTA-PBS containing $3.5 \%$ polyethylene glycol $6000, \mathrm{pH} 7.5$, and further incubated at room temperature for $2 \mathrm{hr}$. The supernatant fluids were discarded after centrifugation at 3,000 rpm for $15 \mathrm{~min}$, and the precipitates were counted for radioactivity in an automatic gamma counter. The binding of labeled antigen was estimated after subtracting non-specific binding which was estimated by using NRS-EDTA-PBS instead of diluted antiserum. The binding percentage was calculated with the standard zero as $100 \%$ where no antigen was added.

Non-competitive binding tests

Non-competitive binding experiments were carried out with an incubation system consisting of $400 \mu \mathrm{l}$ of BSA-PBS, $100 \mu \mathrm{l}$ of ${ }^{125}$ I-labeled antigen dissolved in BSA-PBS, and $100 \mu \mathrm{l}$ of NRS-EDTA-PBS or antisera diluted in NRS-EDTA-PBS. The mixture was incubated at room temperature overnight, and added to $250 \mu \mathrm{l}$ of the second antibody, and further incubated at room temperature for $2 \mathrm{hr}$, then centrifuged. The precipitates were counted for radioactivity. The amounts of radioactive antigen bound to the antibody were expressed as percentages of the radioactive antigen added to the incubation system after subtracting the non-specific binding $(4,8)$.

Immunohistochemistry

Rat pituitaries were fixed with Bouin-Hollande-sublimate for 2 days. After dehydration and embedding in paraplast, $4 \mu \mathrm{m}$-thick sections were cut and mounted in gelatin-coated slides. The deparaffinized sections were treated with iodine alcohol and sodium pyrosulfite to eliminate the sublimate, and then rinsed with distilled water, followed by PBS. The sections were immunostained by peroxidase-anti-peroxidase (PAP) method (7) with minor modifications or immunogold silver staining (IGSS) according to Holgate et al. (2).

\section{RESULTS AND DISCUSSION}

\section{Specificity of antisera in RIA}

In RIA, specificity of an antiserum has been examined by using competitive binding test where a fixed amount of labeled antigen and antibody is incubated in the presence of varied concentrations of the substance which is suspected to crossreact 
with the antibody to see if any inhibition of binding of labeled antigen to the antibody occurs or not, and the inhibition curve obtained is compared with the inhibition curve drown with the antigen (i.e. standard curve for RIA).

In this kind of test with the suspected substances, three types of inhibition curves are usually recognized as shown in Fig. 1. They are: 1) inhibition curve parallel to the standard curve, 2) inhibition curve which is not parallel to the standard curve with less steeper slope than standard, and 3) no inhibition, i.e. the relative binding percentage remains around $100 \%$ though the concentration of the substance is increased.

When the inhibition curve with the substance is parallel with the standard curve, the crossreactivity is calculated from the horizontal distance of the two inhibition curves. For example, if $50 \%$ inhibition was obtained at $10 \mathrm{ng} / \mathrm{ml}$ in the standard curve, and at $200 \mathrm{ng} / \mathrm{ml}$ in the case of the substance tested, the crossreaction is calculated to be $5 \%$. In other words, the substance tested would bind which is $1 / 20$ of that of original antigen to the antibody. In RIA, if the crossreactivity is less than $1 \%$, usually the crossreaction is allowed to be ignored. When a parallel inhibition curve is obtained, we have to think another possible situation. An inhibition curve which is parallel to the standard curve is also obtained when the test substance is contaminated with the original antigen. In this case, the apparent crossreactivity reflects the percentage content of the original antigen in the test substance. But anyway, if this amount is less than $1 \%$, this possibility is also ignored because the test substance preparation will influence the assay result by only $1 \%$ even if the same amount of the test substance to the original antigen is present in the assay sample.

The second type of inhibition curve, which is not parallel to the standard cuve, very often happens when the same hormones of the different species are examined, and also when the subunits of the original antigen such as $\alpha$ or $\beta$ subunit of hCG are examined against anti-original antigen. The non-parallelism of the inhibition curve

B/Bo \%

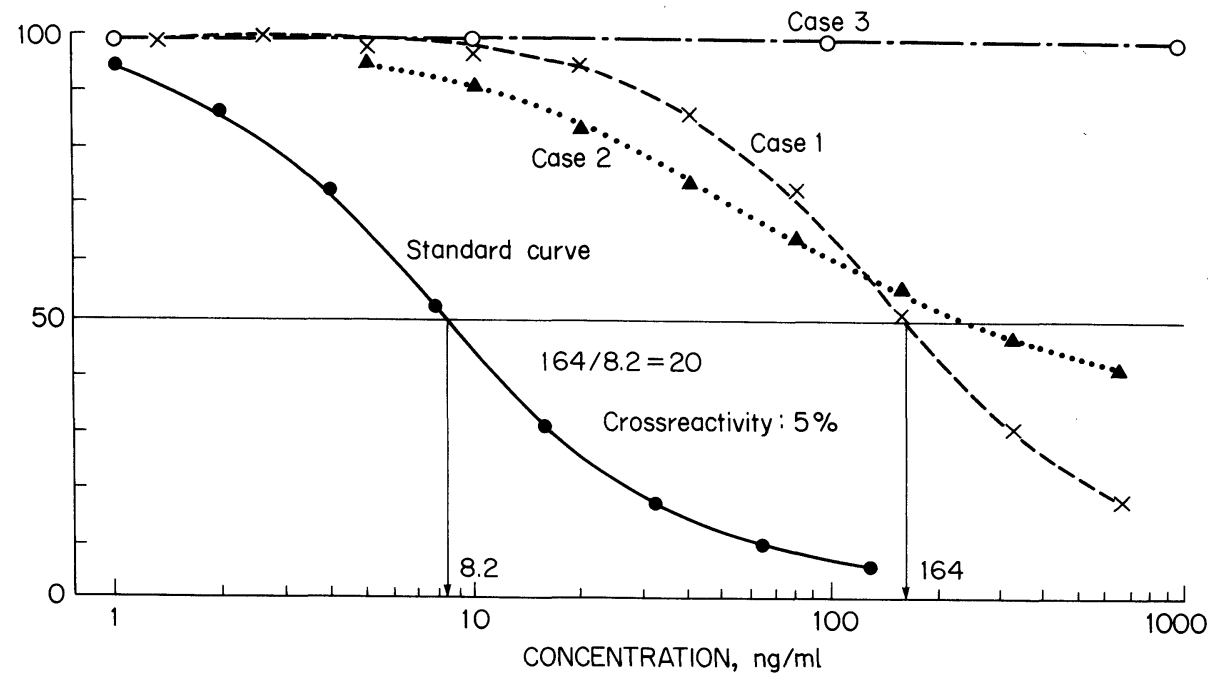

FIG. 1. Model standard curve and inhibition curves in the competitive binding test. Case 1: Inhibition curve parallel to the standard curve. Case 2: Inhibition curve not parallel to the standard curve. Case 3: Inhibition curve with no crossreaction. 
often indicates that some anigenic determinants in the test substance molecule are common to those in the original antigen but that the test substance lacks some determinants which are in the molecule of the original antigen. In other words, it indicates partial crossreactivity. When this type of inhibition curve was obtained, and if the horizontal distance is not big enough, we have to be very careful about the possibility of the presence of the test substance in the assay sample.

The third case where no inhibition is observed indicates no crossreactivity of the substance tested.

In RIA, this kind of assessment of antiserum specificity is practically satisfactory. But this method is not applicable to the antisera to be employed in the immunohistochemistry, because the principle of immunohistochemistry is quite different from that of RIA. The principle of RIA is "competitive binding" where the purified labeled antigen is always present, and the antigen in the assay samples binds to a small amount of antibody in a competitive manner with the labeled antigen, and the reaction is carried out in an antigen-excess state. On the other hand, in immunohistochemistry, the binding reaction is carried out in the presence of an excessive amount of antibody.

\section{Specificity in RIA and immunohistochemistry}

Fig. 2 shows a simulated antigen-antibody binding reaction where a fixed amount of antigen (or crossreacting substance) is reacted with a varied concentration of antibody. Let us postulate the original antigen against which the antiserum is raised with $\mathrm{Kd}$ value of $1 \mathrm{pM}$, and crossreacting substances A, B and C with $\mathrm{Kd}$ of 10,100 and $1000 \mathrm{pM}$, respectively. In RIA, this means that their crossreactivities are 10, 1 , and $0.1 \%$, respectively. When $10 \mathrm{pM}$ of the antigen or crossreacting substances is incubated with $10 \mathrm{pM}$ antibody, about $72 \%$ of the antigen is bound to antibody, while substance $\mathrm{A}, \mathrm{B}$, and $\mathrm{C}$ are bound by 37,8 , and $1 \%$, respectively. If antibody concentration is raised to $100 \mathrm{pM}$, bindings of the antigen, and substances $\mathrm{A}, \mathrm{B}$, and $\mathrm{C}$ are $98,90,48$, and $9 \%$, respectively. By further increase of the antibody concentration to $1000 \mathrm{pM}$ the binding of substance $\mathrm{C}$ would be increased to $50 \%$. This simulation

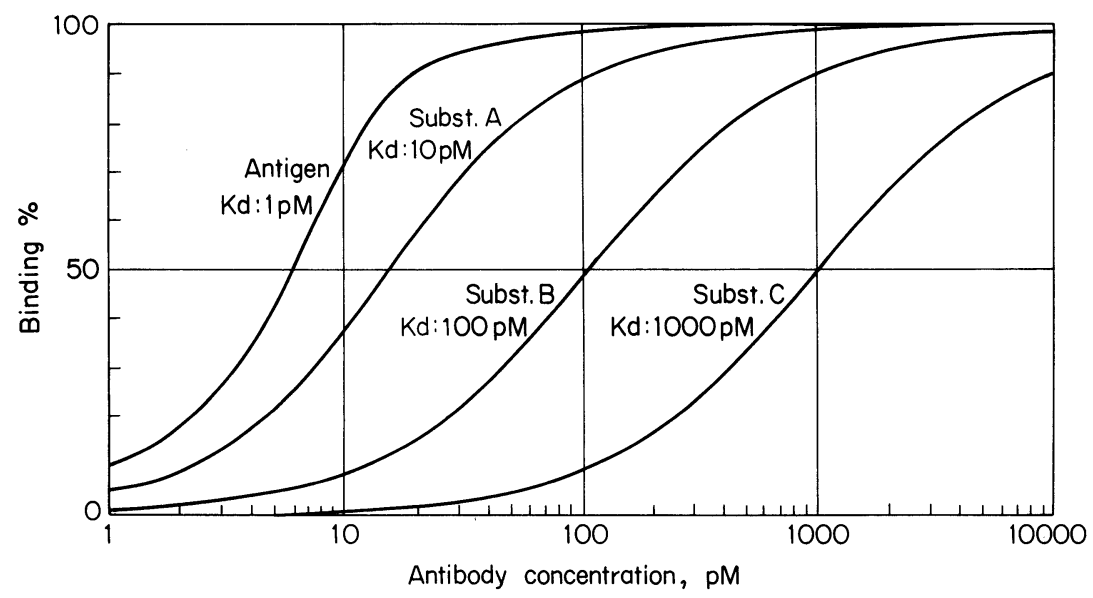

FIg. 2. Simulated binding curves in non-competitive binding test. Curves were drawn with $10 \mathrm{pM}$ of antigen or substances and increasing concentration of antibody. 
indicates that even a substance with very weak affinity can be stained with a higher concentration of antibody.

Fig. 3 shows an example of competitive binding test of anti-hCG. Rat LH shows the crossreactivity less than $0.1 \%$ to anti-hCG and anti-hCG $\beta$. So, no one would use anti-hCG for rat LH RIA. However, in non-competitive binding tests, as shown in Fig. 4, anti-hCG binds about $50 \%$ of labeled hCG at a dilution of $1: 64000$, and binds $50 \%$ of labeled rat LH at 1:8000, while anti-hCG $\beta$ binds $50 \%$ of the labeled hormones at 1:20000 and 1:1500 for hCG and rat LH, respectively. These results indincate that these antisera are useful for immunohistochemical staining of rat LH. Because the similar binding experiments showed that these antisera did not bind other rat pituitary hormones, the staining would be highly specific. Interesting enough, these anti-hCG and anti-hCG $\beta$ showed no significant binding to bovine LH.

A similar example was shown in the previous report of the author about the different nature of mammalian LHRH and avian LHRH (1) where we showed positive immunohistochemical staining of chicken hypothalamus using an antiserum raised against mammalian LHRH though we could not measure avian hypothalamic LHRH by RIA system for mammalian LHRH.

The fact that a substance with a very low affinity to antibody can be bound by using higher concentration of antibody is a merit of immunohistochemistry, and it enables wider application of antibody to various species of animals. But we have to think also that the specificity of immunohistochemical staining is not so strict as RIA, and we should always be careful of staining some other substances which are not intended.

Thus, we have to think that the crossreactivity calculated from RIA inhibition curve can not be applied to immunohistochemistry. The stainability in immunohistochemistry should be assessed from the non-competitive binding tests.

As stated aboved, in RIA system, we cannot distinguish crossreaction from contamination of original antigen in the test substance. Such contamination of antigen can be easily recognized by non-competitive binding test. When the test substance preparation is radioiodinated, the original antigen contaminated in the same preparation is also labeled, and when the labeled preparation is incubated with the antiserum, the contaminated labeled antigen binds to the antibody. However, the antibodybound radioactivity will not exceed the percentage of the contaminant even when the concentration of antibody is increased.

In non-competitive binding test, the labeling of the antigen should be carried out with care not to give critical damage to the binding ability of the antigen to the antibody. Especially with a monoclonal antibody, radioiodination of the tyrosine or histidine which is included in the antigenic determinant causes a serious loss of its antibody binding ability (6).

\section{Presence of antibodies to other substances in antiserum}

Besides the crossreaction, we have to consider the possible presence of antibodies to other substances in the antiserum. In the case of monoclonal antibody, there is no such possibility, however, when we immunize animals with an antigen which is extracted from a tissue, we very often obtain antisera which contain, besides the antibody against the target antigen, antibodies to other substances which are the components of the tissue and are present in very small amount in the antigen preparation. In RIA, this is not a critical problem at all as long as highly pure labeled antigen 


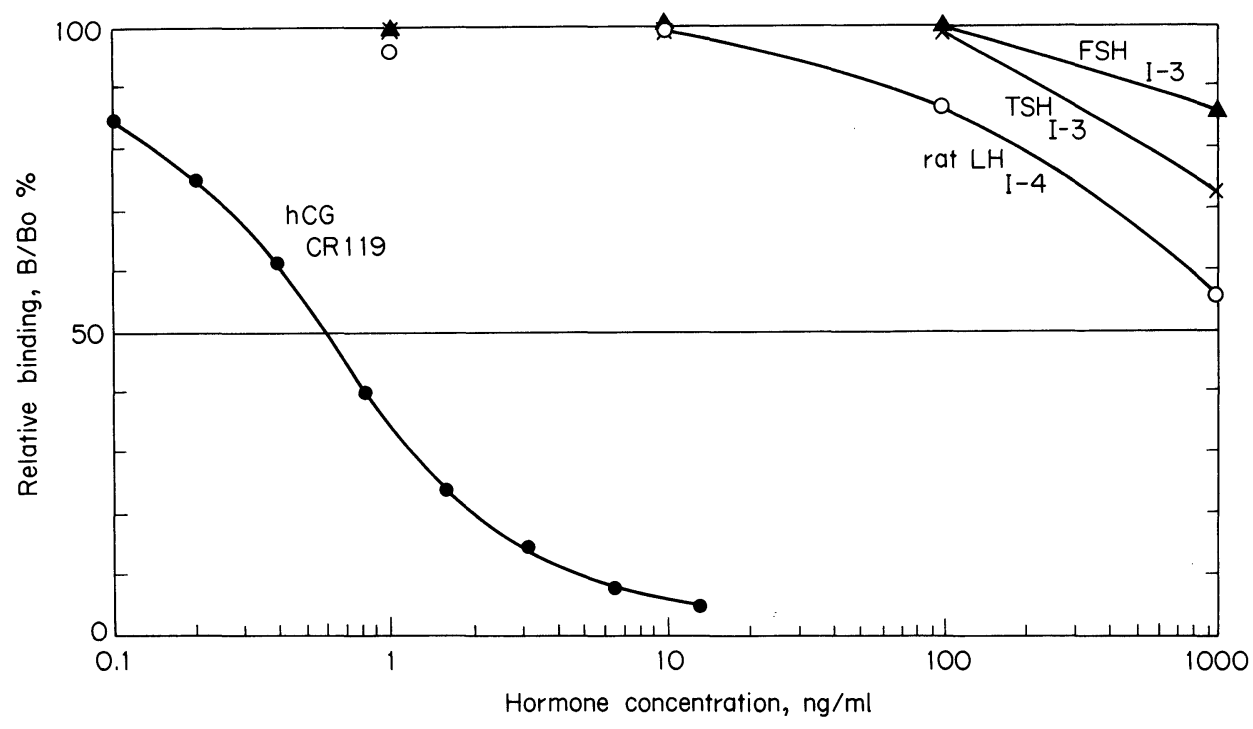

FIG. 3. The competitive binding test of anti-hCG antiserum. As the standard substance, hCG CR-119 was used. The test substances were NIDDK rat LH I-4, rat FSH I-3, and rat TSH I-3.

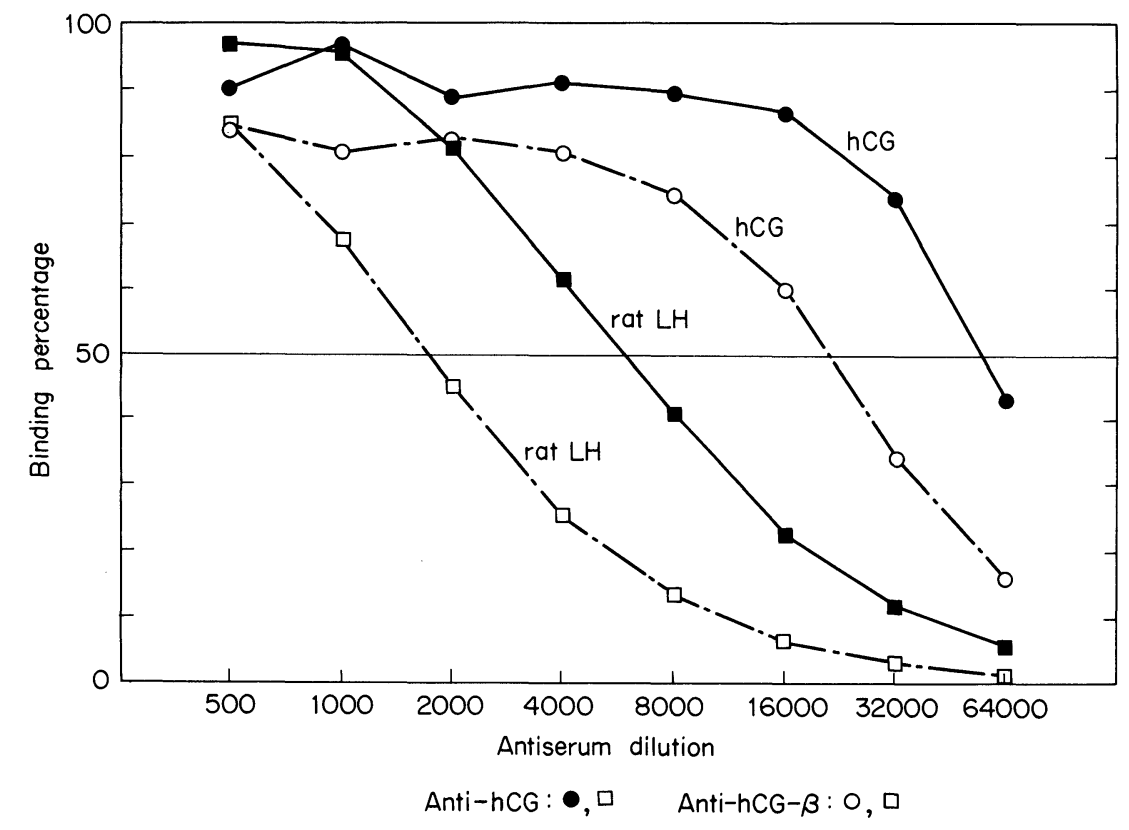

FIG. 4. The non-competitive binding test of anti-hCG and anti-hCG $\beta$ antisera. The labeled substances were hCG CR-119 and NIDDK rat LH I-4. 


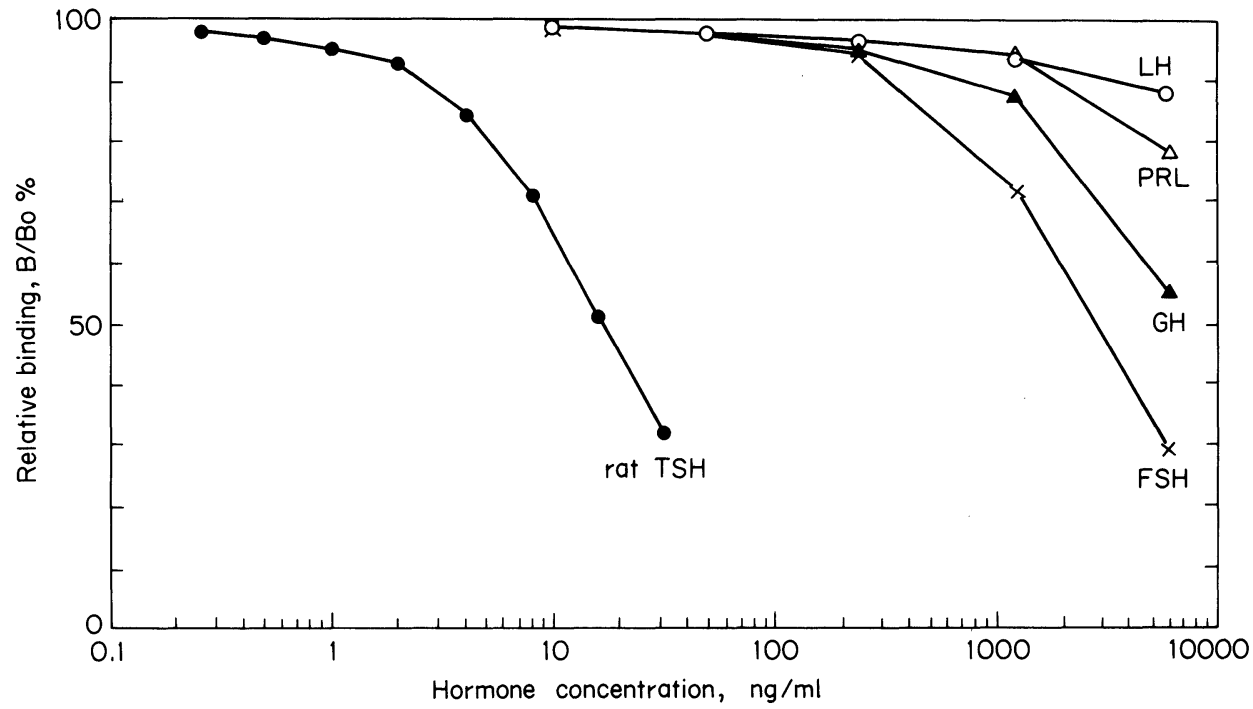

FIG. 5. The competitive binding test of anti-rat TSH serum. The standard and test substances were NIDDK rat TSH I-3, rat LH I-4, rat FSH I-3, rat Prolactin I-3, and rat GH I-3.

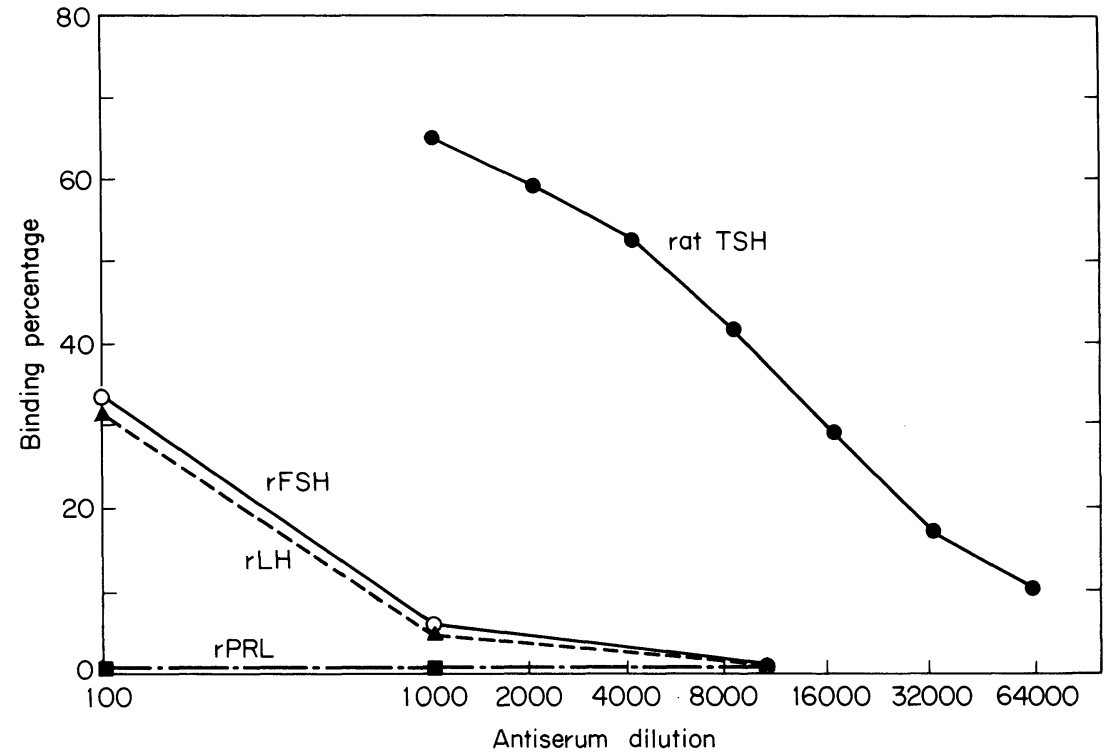

FIG. 6. The non-competitive binding test of anti-rat TSH serum. The labeled substances were NIDDK rat TSH I-3, rat LH I-4, rat FSH I-3, and rat prolactin I-3. 
is used, and by competitive binding test, such presence of antibodies to other substance cannot be found. An example is shown in Figs. 5 and 6 which are the results of competitive and non-competitive binding tests carried out with anti-rat TSH. The competitive binding test shown in Fig. 5 indicates that apparent crossreactivities of rat FSH, GH, PRL, and LH, which are NIDDK preparations of radioiodination grade, are $1.25,0.24,0.1$, and $0.06 \%$, respectively. From the non-competitive binding test shown in Fig. 6, it is indicated that this anti-TSH serum did not bind prolactin at all even at a dilution of 1:100, while it binds LH significantly at dilutions 1:1000 to nearly the same extent to FSH though the crossreactivity of LH is far smaller than that of FSH. So, this binding may be due to anti-LH antibody present in the antiserum. Table 1 shows the results of immunohistochemical staining of rat pituitary thyrotrophs and gonadotrophs. When PAP method was used, this anti-TSH serum gave positive staining of both thyrotrophs and gonadotrophs until the dilution 1:4000 though the grade of staining is different, and at 1:8000 stained only TSH. In the case of IGSS method, this stained only thyrotrophs at 1:30,000. Of course, such a presence of antibodies to other substances can be eliminated by absorption of the antiserum with those substances. This elimination absorption should be carried out, if possible, using immobilized absorbents.

In conclusion, the authors would like to insist that immunohistochemists should not rely solely upon the data from competitive binding test the principle of which is quite different from that of immunohistochemistry. Of course, this kind of test is useful for obtaining the ratio of association or dissociation constants $(\mathrm{Ka}, \mathrm{Kd}$, respectively) if the test substances are highly pure or the largest estimation of the crossreactivity when the test substances are not highly pure. But even when the $\mathrm{Ka}$ is very small (or $\mathrm{Kd}$ is very large), such substance can be stained if higher concentrations of antibody are used. This estimation can be made if one use the non-competitive bin-

TABLE 1. Stainability of anti-rat TSH

\begin{tabular}{ccccccc}
\hline \multirow{2}{*}{$\begin{array}{c}\text { DIlution of } \\
\text { antiserum }\end{array}$} & \multicolumn{2}{c}{ PAP mthod } & & \multicolumn{2}{c}{ IGSS method } \\
\cline { 2 - 3 } \cline { 5 - 6 } $1,000 \times$ & TSH & GTH & & TSH & GTH \\
$2,000 \times$ & ++ & + & & ++ & + \\
$4,000 \times$ & ++ & + & & & ++ & + \\
$8,000 \times$ & ++ & - & + & & ++ & + \\
$16,000 \times$ & + & - & & ++ & + \\
$20,000 \times$ & - & & & + & + \\
$30,000 \times$ & & & & + & + \\
$40,000 \times$ & & & & + & + \\
$80,000 \times$ & & & & + & - \\
\hline
\end{tabular}

\footnotetext{
Fixation: Bouin-Hollande-Sublimate PAP method 1st antibody: overnight IGSS method 2nd antibody: $2 \mathrm{hr}$ 1st antibody: overnight Immunoglobulin-gold complex: $2 \mathrm{hr}$ PAP complex: $2 \mathrm{hr}$ IGSS reaction time: $5 \mathrm{~min}$

$\mathrm{DAB}$ reaction: $2-3 \mathrm{~min}$
} 
ding test because it is based on a similar principle to immunohistochemistry. Another method of non-competitive binding test, the Western blotting method which includes electrophoretic separation is also useful for the assessment of specificity though there is some problem about quantitation (5). The possibility of the presence of antibody against substances other than target antigen can be also clarified if the results of noncompetitive binding test is compared with the results of competitive binding test.

\section{ACKNOWLEDGEMENTS}

The authors are much grateful to Dr. A. F. Parlow of Pituitary Hormones and Antisera Center, Harbor-UCLA Medical Center, and the National Hormone and Pituitary Program, University of Maryland School of Medicine, for their kind supply of NIDDK immuno-reagents.

This work was supported by Grant-in-aid for Developmental Scientific Research No. 60890003 from the Ministry of Education, Science and Culture, Japan.

\section{REFERENCES}

1. Hattori, M., Wakabayashi, K. and Nozaki, M.: Difference of Japanese quail LH-RF from mammalian LH-RF revealed by biological and immunochemical studies. Gen. Comp. Endocrinol. 41; 217-224, 1980.

2. Holgate, C. S., Jackson, P., Cowen, P. N. and Bird, C. C.: Immunogold-silver staining: New method of immunostaining with enhanced sensitivity. J. Histochem. Cytochem. 31; 938-944, 1983.

3. Hunter, W. M. and Greenwood, F. C.: Preparation of iodine-131 labelled human growth hormone of high specific activity. Nature 194; 495-496, 1962.

4. Kawarai, Y.: Identificatioin of ACTH cells and TSH cells in rat anterior pituitary with the unlabeled antibody enzyme method on adjacent thin and thick sections. Acta histochem. cytochem. 13; 627-645, 1980.

5. Park, M. K., Tanaka, S., Hayashi, H., Hanaoka, Y., Wakabayashi, K. and Kurosumi, K.: Production and characterization of a monoclonal antibody against the $\beta$-subunit of bullfrog lutropin. Gen. Comp. Endocrinol. 68; 82-90, 1987.

6. Park M. K. and Wakabayashi, K.: Preparation of monoclonal antibody to common amino acid sequence of LHRH and its application. Endocrinol. Japon. 257-272, 1986.

7. Sternberger, L. A., Hardy, P. H. Jr., Cuculis, J. J. and Mayer, H. G.; The unlabeled antibody enzyme method of immunocytochemistry. Preparation and properties of soluble aitnigen-antibody complex (horseradish peroxidase-antihorseradish peroxidase) and its use in identification of spirochetes. J. Histochem. Cytochem. 18; 315-333, 1970.

8. Tanaka, S. and Kurosumi, K.: Differential subcellular localization of ACTH and $\alpha$-MSH in corticotropes of the rat anterior pituitary. Cell Tissue Res. 243; 229-238, 1986. 